\title{
ELETROCHALLENGE: PROJETO DE EXTENSÃO COM PROPOSTA DE DESAFIO MANTÉM CALOUROS DAS ENGENHARIAS EM CONTATO COM CONTEÚDOS DO CURSO EM TEMPOS DE PANDEMIA
}

\section{DOI: 10.37702/2175-957X.COBENGE.2021.3647}

Edson Cordeiro do Valle - edsoncv@gmail.com Universidade Federal do Rio Grande do Sul Rua Luiz Englert S/N S/N 94040-040 - Porto Alegre - RS

Alberto Bastos do Canto Filho - alberto.canto@ufrgs.br Universidade Federal do Rio Grande do Sul Av. Osvaldo Aranha 103 90035-190 - Porto Alegre - RS

Roberto Petry Homrich - roberto.homrich@ufrgs.br Universidade Federal do Rio Grande do Sul Rua Duque de Caxias 586 90010-280 - Porto Alegre - RS

Luiz Tiarajú dos Reis Loureiro - tiaraju03@gmail.com Universidade Federal do Rio Grande do Sul Av. Osvaldo Aranha 103 90035-190 - Porto Alegre - RS

Dionatan Rafael Argenta de Queiroz - dionatanargenta@gmail.com Universidade Federal do Rio Grande do Sul Rua da Palma 87 93025-320 - São Leopoldo - RS

Laura Gonçalves Patricio - laurapatricio99@hotmail.com Universidade Federal do Rio Grande do Sul Rua das Caravelas 774 91370-160 - Porto Alegre - RS

Marcello Reis Severo - marcellosevero_@hotmail.com Universidade Federal do Rio Grande do Sul 
Rua Domingos Crescêncio 559

90650-090 - Porto Alegre - RS

Romeu Longo Malinski - romeumalinski@gmail.com

Universidade Federal do Rio Grande do Sul

Rua Tupanciretã 140

93334-480 - Novo Hamburgo - RS

Daniel Santana Ribeiro Martins - danielmartins_@live.com

Universidade Federal do Rio Grande do Sul

José Madrid 145

91430-580 - Porto Alegre - RS

Enzo Victor Zucchetti Pietta - enzo.victor@hotmail.com

Universidade Federal do Rio Grande do Sul

Anacleto Peccini 487

95350-000 - Nova Araçá - RS

Caio Henrique Grazzi Bertini Belle - caiobelle8@gmail.com

Universidade Federal do Rio Grande do Sul

Rua João Ronchesel 57

17210-700 - Jaú - SP

Resumo: Durante o período de paralisação das aulas da Universidade Federal do Rio Grande do Sul (UFRGS) devido à pandemia do COVID-19, um projeto de extensão foi proposto aos calouros dos cursos de engenharia. O projeto foi designado Eletrochallenge, cujo objetivo era desenvolver hábitos de estudo, promover interação e motivar os calouros, reduzindo os índices de reprovações e evasões na Universidade. Foram realizadas atividades em grupos, com temáticas relacionadas à engenharia. O projeto foi desenvolvido por 8 estudantes de graduação de engenharia. Foram assessorados por 3 professores orientadores, porém a elaboração das atividades, dinâmicas e administração ficaram sob responsabilidade dos discentes. $O$ projeto foi realizado virtualmente, possibilitando a utilização de atividades didáticas através de plataformas não usualmente empregadas nos cursos da Universidade. Após 4 semanas de atividades, um formulário de feedback foi enviado aos participantes. As respostas mostraram que muitos inscritos no projeto o aprovaram. Dentre os resultados positivos, pode-se mencionar o aprendizado do conteúdo e o aumento na motivação para continuar cursando engenharia. Ademais, muitos relataram que acreditam que foi uma boa 


\section{CCOBENGE

experiência. Os estudantes coordenadores do projeto também desenvolveram competências com o projeto. Habilidades de trabalho em equipe $e$ responsabilidade foram desenvolvidas e aprimoradas. Com o término dos trabalhos e o sucesso do Eletrochallenge, o projeto foi apresentado a autoridades da Universidade, que demonstraram interesse em sua continuidade.

Palavras-chave: Ensino. Projeto. Calouros. Engenharia. Competição. 


\section{ELETROCHALLENGE: PROJETO DE EXTENSÃO COM PROPOSTA DE DESAFIO MANTÉM CALOUROS DAS ENGENHARIAS EM CONTATO COM CONTEÚDOS DO CURSO EM TEMPOS DE PANDEMIA}

\section{INTRODUÇÃO}

\subsection{Contexto}

No principal ranking mundial de educação, o Programa Internacional de Avaliação dos Estudantes (Pisa), é sempre possível encontrar o Brasil nas últimas colocações, muito distante de nações referência em educação básica. Esse mau desempenho escolar se torna ainda mais evidente nas áreas de matemática e ciências, constantemente apresentando resultados abaixo da média se comparado a outros países da Organização para Cooperação e Desenvolvimento Econômico (OCDE). Isso é um claro reflexo da educação básica precária a qual os jovens brasileiros estão submetidos. Uma parcela significativa dos estudantes de graduação das engenharias reprova nas disciplinas iniciais do curso, como as de Cálculo e Física. Isso decorre, em sua maioria, da grande dificuldade dos alunos em ciências exatas, devido a lacunas de formação (LODER, 2009), trazidas desde o ensino médio. Índices elevados de reprovação provocam resultados indesejáveis, como o aumento da demanda de recursos, como salas de aulas e professores para lecionar novamente a disciplina aos estudantes, e aumento da evasão (CANTO, 2015). Sendo assim, medidas para evitá-las são desejadas. É sugerido que hábitos de estudo, associados a outros fatores, como motivação e habilidades de estudo, desempenham um papel crítico e central na determinação do desempenho acadêmico dos estudantes (CREDÉ et al, 2006). O desenvolvimento de hábitos de estudo, todavia, não é incentivado, muitas vezes, pelos currículos universitários. Se isso fosse promovido, é possível que o número de reprovações e evasões diminuísse. A colaboração e a amizade entre estudantes de graduação, principalmente nos cursos de engenharia, é um fator de grande relevância para a permanência do estudante no curso. Isso é acreditado levando em consideração a dificuldade elevada dos cursos de engenharia e o consequente estresse provocado nos estudantes, sendo as amizades uma forma de lidar com tal problema (BUOTE et al., 2007).

\subsection{Proposta}

No início de março, no primeiro semestre de 2020, o mundo começou a sofrer com a pandemia do COVID-19. Em todo o planeta, governos se mobilizaram para conter a rápida disseminação do vírus, visando evitar o colapso dos sistemas de saúde públicos. No Brasil, as instituições de ensino foram as primeiras a cancelar as atividades correntes, como aulas e demais atividades presenciais. A Universidade Federal do Rio Grande do Sul (UFRGS), no dia 15 de março, suspendeu todas as aulas presenciais da graduação, da pós-graduação e do Colégio de Aplicação. O retorno das atividades normais estava previsto para três semanas após anunciada a suspensão, entretanto o período de paralisação foi estendido. No final deste mesmo mês, os professores orientadores apresentaram uma proposta para os alunos de elaborar uma atividade para ser realizada no período de suspensão das aulas presenciais na Universidade. A ideia era de realizar um projeto de extensão, em forma de gincana EAD, em grupos, utilizando o Moodle como 
plataforma para a realização de questionários. As atividades abordariam os conteúdos das disciplinas iniciais do curso, como física, cálculo e introdução à engenharia. Foi decidido que a atividade seria destinada a estudantes das etapas iniciais de cursos relacionados a Engenharia Elétrica. Desta forma, foi decidida a realização de um projeto de extensão denominado Eletrochallenge, tendo em vista que se trataria de uma atividade com enfoque aos cursos relacionados a Engenharia Elétrica, e seria desenvolvido em forma de gincana em grupos, composta por desafios diários. Entretanto, com a divulgação da ideia do projeto com professores do Departamento de Engenharia Química (DEQUI), foi decidido que seriam abertas as inscrições aos calouros de Engenharia Química e que, com isso, atividades de química também seriam incluídas.

\subsection{Objetivos}

O momento de crise em 2020 foi oportuno para a execução deste projeto, visto que a grande maioria dos estudantes nesse período encontrava-se em casa, sem exercer atividades relacionadas aos cursos em que estavam matriculados. Como, no início do período de cancelamento das aulas, a Universidade não autorizava a realização de aulas nem na modalidade remota, os alunos estavam sem contato com o meio acadêmico e tinham a sensação de que estavam de férias. O objetivo principal do Eletrochallenge foi o de incentivar os inscritos a estudarem durante o período de paralisação das aulas da Universidade. Além disso, o desenvolvimento de hábitos de estudo nos calouros, a interação entre os estudantes e a motivação para o aprendizado de engenharia também foram propostos. Essas metas foram definidas tendo em vista que poderiam ser benéficas, a longo prazo, para a Universidade, pois teriam a capacidade de reduzir evasões e reprovações nos cursos de engenharia, minimizando os problemas decorrentes disso. Ademais, o desenvolvimento de competências de aprendizagem autônoma, decorrentes de hábitos de estudo, e do trabalho em grupo, proveniente da interação com colegas, são importantes para o mercado de trabalho e para o aprimoramento pessoal.

\section{METODOLOGIA}

O projeto Eletrochallenge foi desenvolvido como uma atividade de extensão coordenada por professores da Escola de Engenharia da UFRGS com o objetivo de: 1) manter os estudantes ativos e motivados durante o período de suspensão das aulas devido à pandemia COVID-19; 2) desenvolver competências gerenciais dos estudantes participantes da equipe de projeto; 3) estimular os calouros a desenvolver hábitos de estudos diários.

\subsection{A Equipe Organizadora}

A Equipe Organizadora foi composta por oito estudantes de engenharia, dos cursos de Engenharia Elétrica, Engenharia Química e Engenharia de Controle e Automação.

Esta equipe recebeu orientações gerais sobre as datas e o período de realização do projeto e as seguintes diretrizes:

1. Desenvolver um projeto que tenha por objetivo a motivação e o aprendizado dos calouros durante o período de suspensão das aulas;

2. O projeto deveria ser uma competição por equipes;

3. As regras da competição devem privilegiar as equipes nas quais os os estudantes com menor preparo tenham oportunidade de aprender com os colegas. 
Dentro destas diretrizes, a Equipe Organizadora teve ampla liberdade para: 1) propor o regulamento; 2) propor as atividades e tarefas integrantes da competição; 3) gerenciar o projeto como um todo.

Sempre que necessário, a Equipe Organizadora se reunia para realizar o planejamento e ajustes necessários, tais como discutir as tarefas dos próximos dias e resolver problemas ocorridos durante a competição.

A interação com os professores orientadores ocorreu através de uma reunião semanal de acompanhamento de projeto ou através de reuniões agendadas sempre que houvesse uma demanda específica.

\subsection{Dinâmica do desafio}

Após o término das inscrições grupos foram organizados de forma mais heterogênea possível, ou seja, com integrantes de todos os cursos inscritos. Isso foi feito para possibilitar a interação dos participantes com colegas de outros cursos. Logo é de grande importância a troca de conhecimentos entre os alunos de diferentes formações.

A competição teve duração de quatro semanas. Todos os dias, atividades eram divulgadas e os grupos precisavam realizá-las para pontuarem. As tarefas foram, em sua maioria, questionários realizados em diferentes tipos de plataforma.

As questões propostas foram desenvolvidas e escolhidas pela Equipe Organizadora, desta forma desenvolvendo a habilidade criativa dos organizadores do Eletrochallenge, já que a proposta era de realizar questões temáticas sobre engenharia.

As atividades individuais deveriam ser realizadas por todos os estudantes e definiriam a nota individual e da equipe no projeto. Assim, conceitos diferentes poderiam ser obtidos por participantes de uma mesma equipe. Isso foi pensado desta forma para motivar o estudo de todos os inscritos.

\subsection{Conteúdos abordados}

A maioria dos conteúdos abordados deveriam ser de nível médio. Entretanto, como forma de introduzir conteúdos universitários, algumas noções básicas de ensino superior também foram incluídas. Além disso, o desafio também visava desenvolver a capacidade de aprender autonomamente, sendo essa habilidade essencial para os acadêmicos de cursos de engenharia. Disciplinas do ensino superior como Cálculo I, Física I, Introdução à Engenharia, Geometria Descritiva, Introdução a Programação e Química Geral foram os conteúdos abordados no evento.

\subsection{Atividades}

As atividades de modo geral foram tarefas que se tratavam de questionários de com 10 questões, com algumas horas de duração. Dentre essas questões, 7 eram as mesmas para todos os estudantes, e 3 eram aleatórias. Isso foi feito para evitar que participantes copiassem as respostas de outros integrantes do evento.

Tarefas e atividades interativas por meio de redes sociais ou até mesmo plataformas externas, foram exploradas no decorrer do evento, pontuaria a equipe que fosse mais rápida e soubesse trabalhar melhor em grupo para encontrar a solução dos desafios propostos.

\section{PLATAFORMAS UTILIZADAS}


Por se tratar de uma atividade na modalidade EAD, devido a pandemia que ocorria concomitantemente, diversas plataformas eletrônicas foram utilizadas para a realização do projeto. A entrega e a realização de tarefas ocorreram por meio de redes sociais, plataformas usuais do meio acadêmico como o Moodle e outras plataformas externas como o Kahoot. Cada site teve sua funcionalidade e importância para o funcionamento do projeto de extensão.

\subsection{Redes Sociais}

O Facebook e o Instagram foram as redes sociais eleitas para serem o principal meio de comunicação entre os integrantes da Equipe Organizadora e os participantes da gincana. Essas mídias foram propostas devido ao grande alcance e à fácil utilização. Nessas plataformas, a pontuação das equipes e alertas de novas atividades foram divulgadas.

Como redes sociais fazem parte do cotidiano de muitos jovens, e para agregar um maior dinamismo ao desafio, algumas tarefas foram realizadas nessas plataformas. Essas atividades foram denominadas "Questões da Página" e consistiam, principalmente, em uma questão de alto grau de complexidade, que precisaria ser respondida pela equipe. Nesse tipo de tarefa, somente a equipe que respondesse primeiro à questão ganharia pontos. Posteriormente, isso foi alterado para pontuar mais de uma equipe por questão, acirrando ainda mais disputa.

\subsection{Moodle}

Essa plataforma educacional foi eleita devido à grande versatilidade e personalização que é possível preparar e realizar questionários. O Moodle, além disso, é a plataforma oficial da UFRGS para cursos EAD, desta forma, seu uso no Eletrochallenge foi uma introdução e proporcionou experiência para o uso futuro do site.

Os organizadores do evento tentaram explorar o máximo de recursos do Moodle, aplicando questionários com exercícios de respostas numéricas, calculadas, de múltipla escolha, e dissertativas. As tarefas neste site deveriam ser realizadas por todos os participantes e contavam presença no projeto de extensão.

\subsection{Kahoot}

A plataforma de aprendizagem baseada em jogos foi de grande importância, pois possibilitou a realização de questionários de múltipla escolha mais descontraídas, com menor dificuldade, entretanto com menor tempo de resposta do que o Moodle. Esta plataforma conta com uma interface interativa bem amigável, com músicas de fundo e menus coloridos.

Geralmente, às sextas-feiras, eram realizadas as atividades nesta plataforma. A pontuação dos questionários dependia do tempo de resposta de cada questão e a quantidade de acertos consecutivos, premiando, assim, grupos que respondiam com maior rapidez.

\section{SUPORTE AO APRENDIZADO AUTÔNOMO}

Um dos objetivos do projeto era o de incentivar o estudo contínuo dos estudantes, mesmo no período de cancelamento das aulas na Universidade devido à pandemia. Isso 
foi concretizado por meio de atividades diárias que necessitavam um estudo prévio do assunto tratado para a obtenção de bons resultados.

Como forma de disponibilizar materiais de estudo aos participantes, uma parceria com uma plataforma de estudos on-line foi feita. Foi decidido, por meio de reuniões e troca de mensagens, que o site disponibilizaria gratuitamente videoaulas, exercícios e demais materiais dos conteúdos abordados no desafio, especificados no regulamento.

O suporte ao aprendizado autônomo também foi visto dentro das equipes. Como as notas individuais influenciavam na nota do grupo, era necessário o apoio dos estudantes com facilidade de compreensão para com os estudantes que estivessem com alguma dificuldade nos conteúdos abordados ou com lacunas de formação. Essa dinâmica ainda, como efeito colateral, estimulava habilidades de trabalho em grupo e liderança no projeto, assim como competências de convívio e interação com colegas de trabalho.

\section{RESULTADOS E CONCLUSÕES}

Após 4 semanas de atividades, o projeto foi encerrado. Os objetivos do Eletrochallenge consistiam-se em incentivar o estudo durante o período de paralisação das aulas da Universidade, desenvolver hábitos de estudo nos calouros, promover a interação entre os estudantes e motivar o aprendizado de engenharia.

A interação entre os estudantes, importante para a formação de uma Networking de Estudos e para o desenvolvimento de relações e contatos, foi promovida por meio da dinâmica do projeto ser em grupos. Os participantes, para atingir boas notas e pontuações no Eletrochallenge, precisaram interagir com suas equipes.

Hábitos de estudo também foram desenvolvidos com o projeto, pois, na maioria dos dias, questionários deveriam ser respondidos. O preparo diário para as atividades foi proposto para desenvolver esse hábito positivo nos participantes. Isso é possível de ser percebido quando comparadas as médias do primeiro questionário com os questionários seguintes.

Com a idealização do Eletrochallenge, foi planejada a realização de atividades contextualizadas e temáticas de engenharia. Além disso, por colocar em prática os conteúdos vistos na graduação, os calouros demonstraram motivação para continuar os estudos.

O projeto também foi uma pesquisa de novos métodos de ensino de engenharia, já que atividades de diversos tipos foram realizadas e informações, coletadas. A adaptação de atividades didáticas em um contexto de gincana e competição foi, em parte, responsável pelos resultados atingidos. O pioneirismo proporcionado pelo Eletrochallenge pode ser o ponto de partida para projetos com esses objetivos.

\subsection{Conceitos e notas obtidas}

Ao final do evento, seria oferecido um certificado de atividade de extensão, para aqueles estudantes que obtiveram presença acima de $75 \%$ nos questionários do Moodle, dando direito a horas extras na Universidade. Estavam previstos, no regulamento, os conceitos e suas respectivas faixas de notas: conceito $A$ (média igual ou superior a 8,5 nos questionários Moodle e Kahoot); conceito $B$ (média entre 5,5 e 8,4 nos questionários Moodle e Kahoot); conceito C (média abaixo de 5,5 nos questionários Moodle e Kahoot).

Figura 1 - Gráfico de conceitos obtidos pelos concluintes do Eletrochallenge 


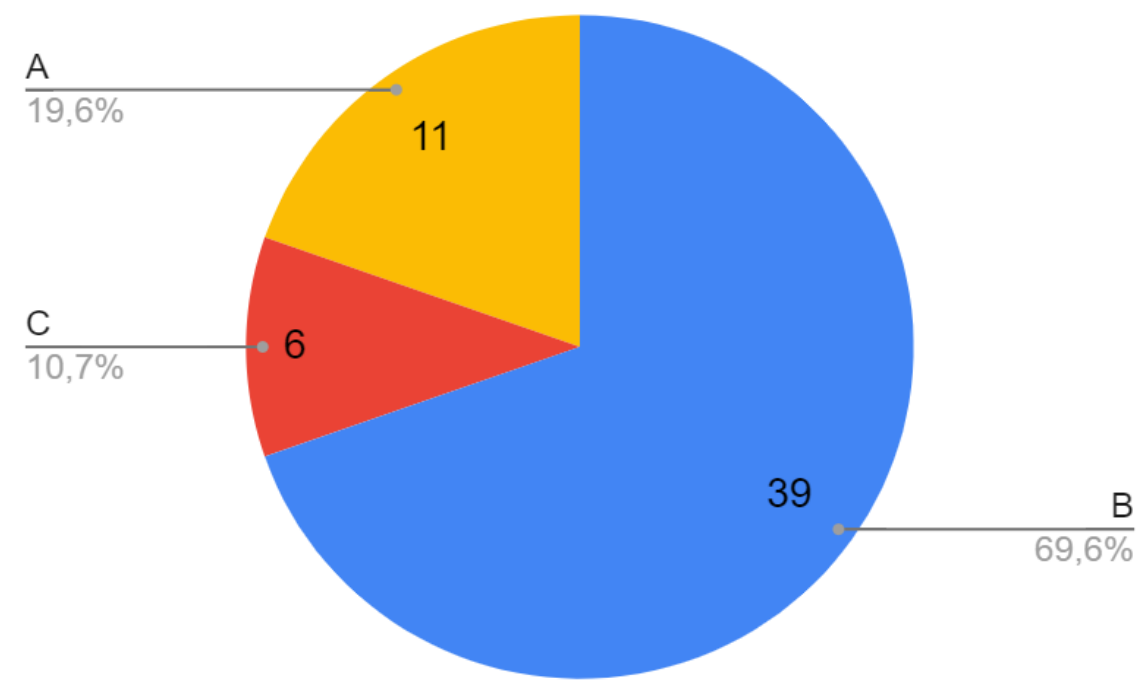

Fonte: Equipe Organizadora do Eletrochallenge, 2020

\subsection{Visão dos participantes}

Ao final do projeto de extensão, um questionário foi disponibilizado aos alunos. Composta por 23 perguntas, a pesquisa visava compreender a visão dos participantes sobre as atividades, a dinâmica do projeto, as equipes e outras informações que poderiam ser úteis para aprimorar o projeto caso fosse realizada uma próxima edição. Ao todo, 35 respostas foram recebidas.

Mais da metade dos indivíduos que responderam o feedback $(62,9 \%)$ disseram que a nota que dariam para experiência que tiveram com o Eletrochallenge seria igual ou maior do que 8, assim como mostra a figura 2. Isso demonstra que os participantes gostaram da dinâmica das atividades e do projeto como um todo.

Figura 2 - Feedback dos participantes

Qual nota você daria para sua experiência no Eletrochallenge?

35 respostas

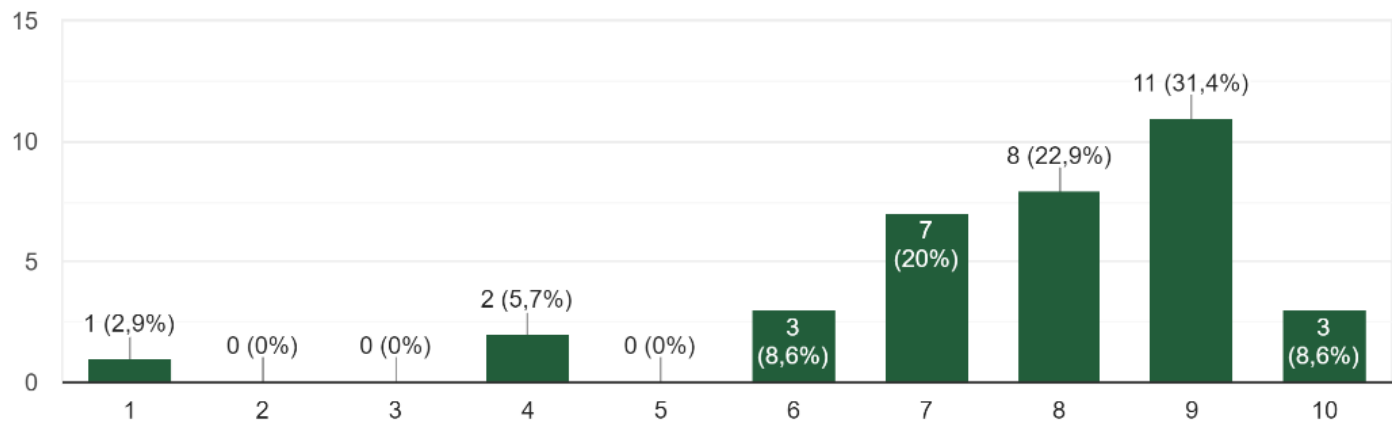

Fonte: Equipe Organizadora do Eletrochallenge, 2020.

\subsection{Visão da Equipe Organizadora}

Os organizadores do Eletrochallenge acreditam que fizeram um trabalho satisfatório em promover os objetivos propostos para o projeto. Além disso, a experiência 
de coordenar e organizar uma atividade desta magnitude proporcionou diversos aprendizados que, de outra forma, seriam difíceis de se obter.

Dentre os aprendizados obtidos, é importante ressaltar o desenvolvimento de habilidades de trabalho em grupo e liderança. Isso se tornou evidente com a divisão de tarefas, principalmente na correção de atividades, tendo em vista que, nesses momentos, era necessário expor suas visões e escutar a perspectivas dos colegas para um consenso ser chegado. Importante lembrar também que, para o funcionamento contínuo das atividades, trabalhos paralelos ocorriam na Equipe Organizadora, como a elaboração de resoluções e a montagem dos questionários. A gerencia de recursos, principalmente 0 tempo, foi desenvolvida desta forma, assim como a habilidade de tomar iniciativa para que ideias propostas fossem implementadas.

As reuniões, que ocorriam três vezes por semana, também possibilitaram experiências em liderança e trabalho em grupo. Como alguns organizadores nunca haviam realizado trabalhos envolvendo uma quantidade grande de colegas, como o Eletrochallenge, competências de convencimento e cooperação precisaram ser desenvolvidas para o funcionamento contínuo do projeto. A capacidade de trabalho em grupo é importante para o mercado de trabalho e para trabalhos acadêmicos nos tempos atuais, ainda mais no meio digital, como foi o caso do Eletrochallenge. $O$ desenvolvimento, logo no início do curso, dessas habilidades pode influenciar positivamente os organizadores em futuros projetos.

Competências de autonomia, responsabilidade e agilidade foram praticadas extensivamente durante a execução do Eletrochallenge, considerando que tomadas de decisões foram deixadas a cargo dos estudantes responsáveis, havendo pouca interferência dos professores envolvidos com o projeto. Logo que ocorriam problemas, como erros de gabarito e enunciados de difícil interpretação, os organizadores procuravam soluções e, rapidamente, as complicações eram resolvidas. Isso foi muito elogiado pelos participantes, pois facilitou a dinâmica da competição.

A utilização de novas plataformas, como o Kahoot, Moodle e Latex, viabilizou o aprendizado destas ferramentas por parte da Equipe Organizadora. Um curso foi disponibilizado aos organizadores para aprenderem a criar questões e questionários no Moodle. Sem o projeto, esse conhecimento provavelmente não seria introduzido aos estudantes tão cedo. O Latex, utilizado para a escrita do gabarito dos questionários, é de extrema importância para estudantes de ciências exatas e engenharias, pois facilita a formatação de equações. O Eletrochallenge foi uma forma de introduzir essa linguagem aos organizadores em um contexto prático e útil.

\subsection{Trabalhos futuros}

No momento das inscrições, o incentivo de professores de todos os departamentos envolvidos poderia aumentar a inclusão de certos cursos, tornando, assim, as equipes mais heterogêneas. No caso desta edição, poucos estudantes de Engenharia de Computação participaram do projeto, pois não houve tanta motivação quanto ocorreu nos cursos de Engenharia Elétrica e Química, mesmo que a quantidade de ingressantes nos cursos sejam similares.

A definição dos horários das atividades antes das inscrições reduziria os problemas relacionados a conflitos de horários. Alguns participantes foram desligados do Eletrochallenge devido a esse fator. O tempo de realização das atividades também deve ser considerado, pois o primeiro questionário teve pouco tempo para a realização de questões complexas, o que desmotivou certos participantes. 
Alterações nos critérios de avaliação devem ser pensadas. Nesta primeira edição, foram consideradas somente as notas das atividades do Kahoot e do Moodle para a atribuição de conceitos. Isso apresentou alguns problemas, já que alguns participantes e grupos deixaram de responder as últimas atividades em grupos, pois não seriam relevantes para a obtenção do certificado. A inclusão de atividades em grupos de grande complexidade, como relatórios e vídeos, na nota individual poderia motivar a realização delas.

Grande parte dos estudantes relataram que gostariam que mais atividades interdisciplinares que colocassem em prática os conhecimentos de engenharia. Isso foi implementado no projeto, todavia a maioria das atividades consistiam-se em questionários. Talvez, adicionar mais dinâmicas de trabalho em grupo e atividades criativas e práticas durante o projeto, além de contextualizar a maioria dos questionários, podem aprimorar as futuras edições.

A inclusão de alunos veteranos é uma possibilidade para as futuras edições do Eletrochallenge, abrangido um escopo ainda maior de atuação e impacto do projeto. Com os aprendizados das edições anteriores, os futuros trabalhos podem ser aperfeiçoados. Desta forma, é esperado que cada edição tenha suas particularidades. Devido ao sucesso do projeto, o Eletrochallenge tem potencial para se tornar uma atividade regular, e assim abre portas para novas metodologias de ensino.

\section{REFERÊNCIAS}

BUOTE, V. M. et al. The Importance of Friends: Friendship and Adjustment Among 1st-Year University Students. Journal of Adolescent Research, v. 22, n. 6, p. 665-689, nov./2007. Disponível em: <https://doi.org/10.1177/0743558407306344>. Acesso em: 15 abr. 2020.

CANTO, A. B. DO. MOTRAC - Um modelo de trajetórias de aprendizagem conceitual. Tese (Doutorado em Informática na Educação) - Programa de Pós-Graduação em Informática na Educação. UFRGS, Porto Alegre, 2015.

CREDÉ, Marcus; KUNCEL, Nathan R.. Study Habits, Skills, and Attitudes: The Third Pillar Supporting Collegiate Academic Performance. Perspectives on Psychological Science, v. 3, n. 6, p. 425-453, nov./2008. Disponível em: <https://doi.org/10.1111/j.17456924.2008.00089.x>. Acesso em: 7 mai. 2020.

INEP. Pisa 2018 revela baixo desempenho escolar em leitura, matemática e ciências no Brasil. Disponível em: http://portal.inep.gov.br/artigo/-/assetpublisher/B4AQV9zFY7Bv/content/pisa-2018-revela-baixo-desempenho-escolar-emleitura-matematica-e-ciencias-no-brasil/21206. Acesso em: 2 abr. 2020.

LODER, L. L. ENGENHEIRO EM FORMAÇÃO: O sujeito da aprendizagem e a construção do conhecimento em engenharia elétrica. UFRGS, Porto Alegre, 2009.

EXTENSION PROJECT KEEPS FUTURE ENGINEERS IN CONTACT WITH THE COURSE CONTENT IN PANDEMIC TIMES

Abstract: During the period of interruption of classes at the Federal University of Rio Grande do Sul (UFRGS) due to the pandemic of COVID-19, an extension project was 
proposed to the freshmen of the engineering courses. The project was called Eletrochallenge, whose objective was to develop study habits, promote interaction and motivate freshmen, reducing the failure and dropout rates at the University. Activities were carried out in groups, with themes related to engineering. The project was developed by 8 engineering undergraduate students. They were assisted by 3 supervising teachers, but the elaboration of activities, dynamics and administration were under the responsibility of the students. The project was carried out virtually, making it possible to use didactic activities through platforms not usually employed in the University's courses. After 4 weeks of activities, a feedback form was sent to the participants. The responses showed that many registrants in the project approved it. Among the positive results, we can mention the learning of the content and the increase in motivation to continue studying engineering. In addition, many students reported that they believe it was a good experience. The project's coordinating students also developed skills with the project. Teamwork and responsibility skills have been developed and improved. With the completion of the work and the success of Eletrochallenge, the project was presented to University authorities, who showed interest in its continuity.

Keywords: Teaching. Project. Freshmen. Engineering. Competition. 